\title{
Spin density wave induced disordering of the vortex lattice in superconducting $\mathrm{La}_{2-x} \mathrm{Sr}_{x} \mathrm{CuO}_{4}$
}

\author{
J. Chang, ${ }^{1,2}$ J. S. White, ${ }^{1,2}$ M. Laver, ${ }^{1,3,4}$ C. J. Bowell, ${ }^{5}$ S. P. Brown, ${ }^{5}$ A. T. Holmes, ${ }^{5}$ L. Maechler, ${ }^{1}$ S. Strässle, ${ }^{6}$ \\ R. Gilardi, ${ }^{1}$ S. Gerber, ${ }^{1}$ T. Kurosawa, ${ }^{7}$ N. Momono, ${ }^{7}$ M. Oda,${ }^{7}$ M. Ido, ${ }^{7}$ O. J. Lipscombe, ${ }^{8}$ S. M. Hayden, ${ }^{8}$ C. D. \\ Dewhurst, ${ }^{9}$ R. Vavrin, ${ }^{1}$ J. Gavilano, ${ }^{1}$ J. Kohlbrecher, ${ }^{1}$ E. M. Forgan, ${ }^{5}$ and J. Mesot ${ }^{1,2}$ \\ ${ }^{1}$ Laboratory for Neutron Scattering, Paul Scherrer Institut, CH-5232 Villigen, Switzerland \\ ${ }^{2}$ Institut de la materière complexe, Ecole Polytechnique Fedédérale de Lausanne (EPFL), CH-1015 Lausanne, Switzerland \\ ${ }^{3}$ Department of Physics, Technical University of Denmark, DK-2800 Kgs. Lyngby, Denmark \\ ${ }^{4}$ Nano-Science Center, Niels Bohr Institute, University of Copenhagen, DK-2100 Kфbenhavn, Denmark \\ ${ }^{5}$ School of Physics and Astronomy, The University of Birmingham, Birmingham B15 2TT, United Kingdom \\ ${ }^{6}$ Physik-Institut der Universitat Zürich, CH-8057 Zürich, Switzerland \\ ${ }^{7}$ Department of Physics, Hokkaido University-Sapporo 060-0810, Japan \\ ${ }^{8}$ H. H. Wills Physics Laboratory, University of Bristol, Bristol, BS8 1TL, United Kingdom \\ ${ }^{9}$ Institut Laue-Langevin, 6 rue Jules Horowitz, 38042 Grenoble, France
}

(Received 23 November 2011; revised manuscript received 6 February 2012; published 18 April 2012)

\begin{abstract}
We use small-angle neutron scattering to study the superconducting vortex lattice in $\mathrm{La}_{2-x} \mathrm{Sr}_{x} \mathrm{CuO}_{4}$ as a function of doping and magnetic field. We show that near optimally doping the vortex lattice coordination and the superconducting coherence length $\xi$ are controlled by a Van Hove singularity crossing the Fermi level near the Brillouin zone boundary. The vortex lattice properties change dramatically as a spin-density-wave instability is approached upon underdoping. The Bragg glass paradigm provides a good description of this regime and suggests that spin-density-wave order acts as a source of disorder on the vortex lattice.
\end{abstract}

DOI: 10.1103/PhysRevB.85.134520

PACS number(s): 74.25.Uv, 74.25.Ha, 74.72.-h

\section{INTRODUCTION}

A commonality across the borocarbides, ${ }^{1-4}$ cuprates, ${ }^{5}$ ferropnictides, ${ }^{6}$ heavy-fermion, ${ }^{7}$ and organic superconductors 8 is the coexistence of magnetism and superconductivity. The corresponding order parameters typically compete ${ }^{9}$ and often a small perturbation is sufficient to tip the balance between the two. For example, the magnetism carried by the rare earth ions $\mathrm{R}$ in the borocarbides $\mathrm{RNi}_{2} \mathrm{~B}_{2} \mathrm{C}$ can lead to nearly reentrant superconducting phase diagrams, and spontaneously forming superconducting vortices at zero applied field. ${ }^{1-4}$ Equally, vortices induced under applied fields may permit enhanced magnetic correlations in the core regions where the superconducting order parameter is suppressed. ${ }^{10-13}$ This idea was put forward to explain field-induced and field-enhanced magnetic correlations observed in the cuprate superconductor $\mathrm{La}_{2-x} \mathrm{Sr}_{x} \mathrm{CuO}_{4}$ (LSCO). ${ }^{5,14}$

Although the effect of static magnetism on moving vortices was recently considered theoretically ${ }_{1}^{15}$ little is known about how the presence of magnetic correlations affects the arrangement of vortices. Here we address the problem from an experimental point of view. When magnetism and superconductivity coexist there are at least four relevant length scales: the penetration depth $\lambda$, the vortex core size $\xi$, the vortex spacing $a_{0}$, and the magnetic correlation length $\zeta$. The vortex spacing $a_{0} \propto H^{-0.5}$ scales with the applied magnetic field $\mu_{0} H$ and in LSCO the magnetic correlation length can be tuned by varying the doping concentration. Using small-angle neutron scattering (SANS) we have studied two different regimes (see Fig. 1); (i) far away from the magnetic ordering where $\xi, \zeta \ll a_{0}$ and (ii) entering the magnetic phase where $\zeta \sim a_{0}$. In the first regime, where static magnetism is absent, the vortex lattice $(\mathrm{VL})$ structure and core size are understood from pure fermiological considerations. In the second regime with static long-range magnetism, the vortex arrangement exhibits increasing disorder. We find these regimes to be well described within the topical Bragg glass paradigm, where disorder results in an algebraic decay of the translational order of the vortices. ${ }^{16,17} \mathrm{VL}$ disordering is usually driven by effects extrinsic to superconductivity such as rare earth magnetism in $\mathrm{RNi}_{2} \mathrm{~B}_{2} \mathrm{C},{ }^{1-4,18-20}$ or sample impurities and crystalline defects. ${ }^{21}$ In contrast, magnetic and $\mathrm{SC}$ order parameters are intertwined in LSCO; we show that this provides a tunable source of VL disorder.

As shown in Fig. 1, the appearance of magnetism is, in essence, concomitant with the suppression of SANS intensity with field and underdoping. Drawing upon results from the literature ${ }^{22-24}$ and observations reported herein, we are also able to plot the VL structure at low temperature $T$ versus magnetic field $H$ and doping $x$.

\section{EXPERIMENTAL METHODS}

Single crystals of $\mathrm{La}_{2-x} \mathrm{Sr}_{x} \mathrm{CuO}_{4}$ with $x=0.105-0.22$ were grown by the traveling solvent floating zone method. ${ }^{25}$ The static and dynamic magnetic properties of the samples were characterized using both neutron diffraction and neutron spectroscopy and good agreement was found between our data ${ }^{26-28}$ and previously published results. ${ }^{5,29-32}$

The SANS experiments reported here were carried out over a series of experiments using the SANS-I instrument at SINQ, ${ }^{36}$ and the D11 and D22 instruments at Institut Laue-Langevin (ILL). In all experiments we adopted the experimental geometry where external magnetic fields $\mu_{0} H$ are applied parallel to the crystal $\mathbf{c}$ axis, and almost parallel to the neutron beam. The scattered neutrons are recorded using a position-sensitive detector placed behind the sample. For each doping, up to three different neutron wavelengths spanning the range $\lambda_{n}=5-16 \AA$ were used in order to cover the applied 


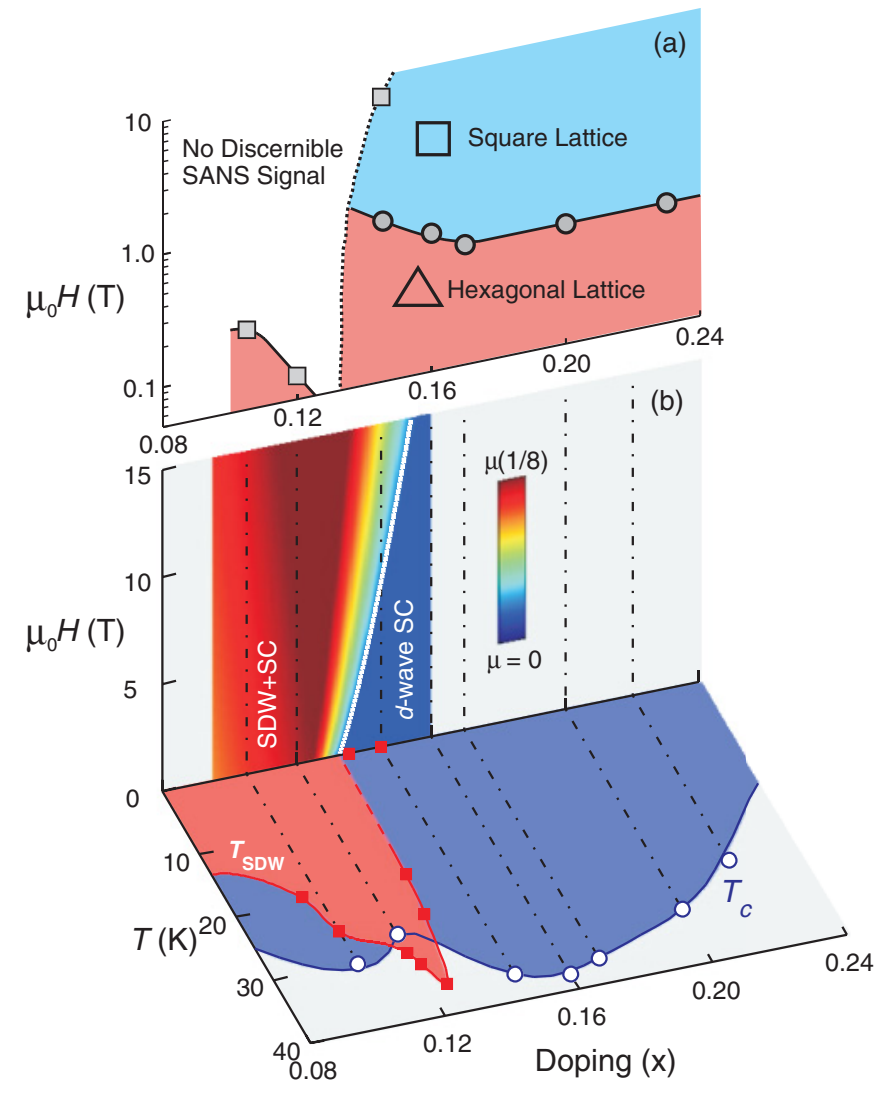

FIG. 1. (Color online) Phase diagram of (a) the $(T<3 \mathrm{~K})$ vortex lattice structure and (b) the magnetism in $\mathrm{La}_{2-x} \mathrm{Sr}_{x} \mathrm{CuO}_{4}$, both revealed by neutron diffraction. Data points in (a) for $x=0.12$, $0.145,0.16$, and 0.22 are from this work whereas $x=0.105,0.17$, and 0.20 are from Refs. 22,23,33. Circular points are defined by the onset of a square VL coordination. The field-doping plane in (b), adapted from Ref. 26, shows schematically the ordered SDW moment normalized to that at the $1 / 8$ doping. Field-induced order was reported by Khaykovich et al. ${ }^{31}$ and later confirmed in Ref. 26. The temperature-doping plane shows the superconducting dome together with the onset of static incommensurate SDW order $T_{\text {SDW }}$ as seen by neutron diffraction. ${ }^{26,34}$ We remark that a similar phase diagram was proposed for $\mathrm{YBa}_{2} \mathrm{Cu}_{3} \mathrm{O}_{y} .{ }^{35}$ The dashed lines indicate the samples studied in this paper.

field $\mu_{0} H$ range of $0.03-10 \mathrm{~T}$. In all cases, a zero-field cooled background was subtracted from the field-cooled data in order to leave just the VL signal.

Our experimental setup is shown in Fig. 2 where we also illustrate the relationship between the VL in the sample and the quantities extracted at the position-sensitive detector. Typically, to observe the signal due to the VL, the sample and cryomagnet are rotated together by angles (such as that shown by $\omega$ in Fig. 2) in order to bring a reciprocal lattice vector onto the Bragg condition at the detector. Due to both the finite resolution of the instrument, and the mosaic spread or imperfection of the VL, the Bragg spots occupy a finite volume in reciprocal space, and can be described, in a first approximation, by three widths $w_{\ell}, w_{\mathrm{q}}$, and $w_{\perp}$ as shown in Fig. 2. These three lengths correspond to the angular widths $\tau_{\omega}, \tau_{\mathrm{r}}$, and $\tau_{\mathrm{A}}$ respectively measured in the SANS experiment. $\tau_{\mathrm{r}}$ and $\tau_{\mathrm{A}}$ describe the finite size of the Bragg

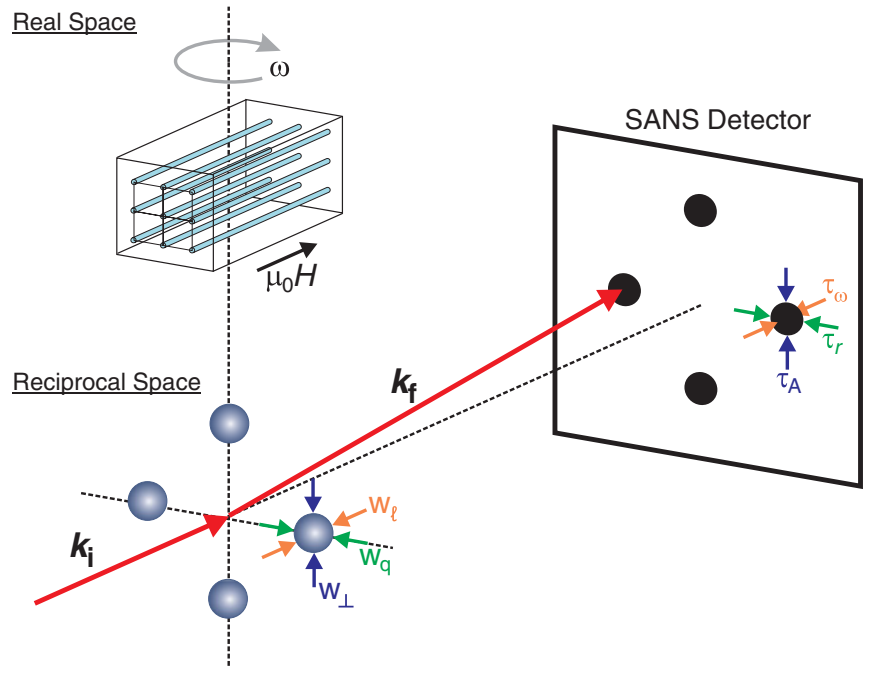

FIG. 2. (Color online) A schematic diagram illustrating the experimental geometry chosen for our experiments. A square VL in real space forms a two-dimensional reciprocal VL, the properties of which are recorded by SANS using a position-sensitive detector. The Bragg spots in reciprocal space exhibit finite widths $w_{\ell}, w_{\mathrm{q}}$, and $w_{\perp}$ that are dependent on both the instrumental resolution and properties of the VL. These three lengths are estimated at the detector by recording the angular widths $\tau_{\mathrm{r}}$ and $\tau_{\mathrm{A}}$ within the detector plane, and $\tau_{\omega}$ perpendicular to the detector plane. $\tau_{\omega}$ is determined experimentally by recording the rocking curve, and corresponds to the rocking curve width.

spot on the detector plane, and $\tau_{\omega}$ is the width of a rocking curve, measured by recording the Bragg spot intensity as a function of rotation angle $\omega$. In the geometry shown in Fig. 2, $\tau_{\mathrm{A}}\left(\right.$ and $w_{\perp}$ ) provide a measure of the VL orientational order about the field axis, while $\tau_{\mathrm{r}}$ (and $w_{\mathrm{q}}$ ) is dominated by the SANS instrumental resolution function. The contribution due to the resolution function is smallest for $\tau_{\omega}$ (and $w_{\ell}$ ), which is sensitive to the VL correlation length along the field direction (vortex straightness). In each of our measurements, the sample and the field were rotated together through a wide range of rocking angles $\omega$ spanning $\pm 5^{\circ}$ about the neutron beam. Note that this angular rotation range is typically narrower than the width of rocking curve in our samples. However, by summing over the measured rocking angles, diffraction patterns such as those shown in Figs. 3(a) and 3(b) are obtained, allowing a determination of the VL properties.

\section{RESULTS AND DISCUSSION}

\section{A. Vortex lattice morphology}

Our observations of the VL structure and coordination can be quantified in terms of a dimensionless parameter $\sigma=4 \pi^{2} \mu_{0} H /\left(\Phi_{0}|\mathbf{G}|^{2}\right)$ where $|\mathbf{G}|$ is the magnitude of the reciprocal VL vector. For a regular hexagonal VL coordination $\sigma=\sqrt{3} / 2$, while for a square coordination $\sigma=1$. The definition of $\sigma$ is useful because it does not require details of the positions $\mathbf{G}$ of Bragg peaks; only the magnitude $|\mathbf{G}|$ is needed. At low fields, the VL is susceptible to orientational disorder due to impurities or defects in the sample, but |G| can still be measured. For example, in Fig. 3(a) the 

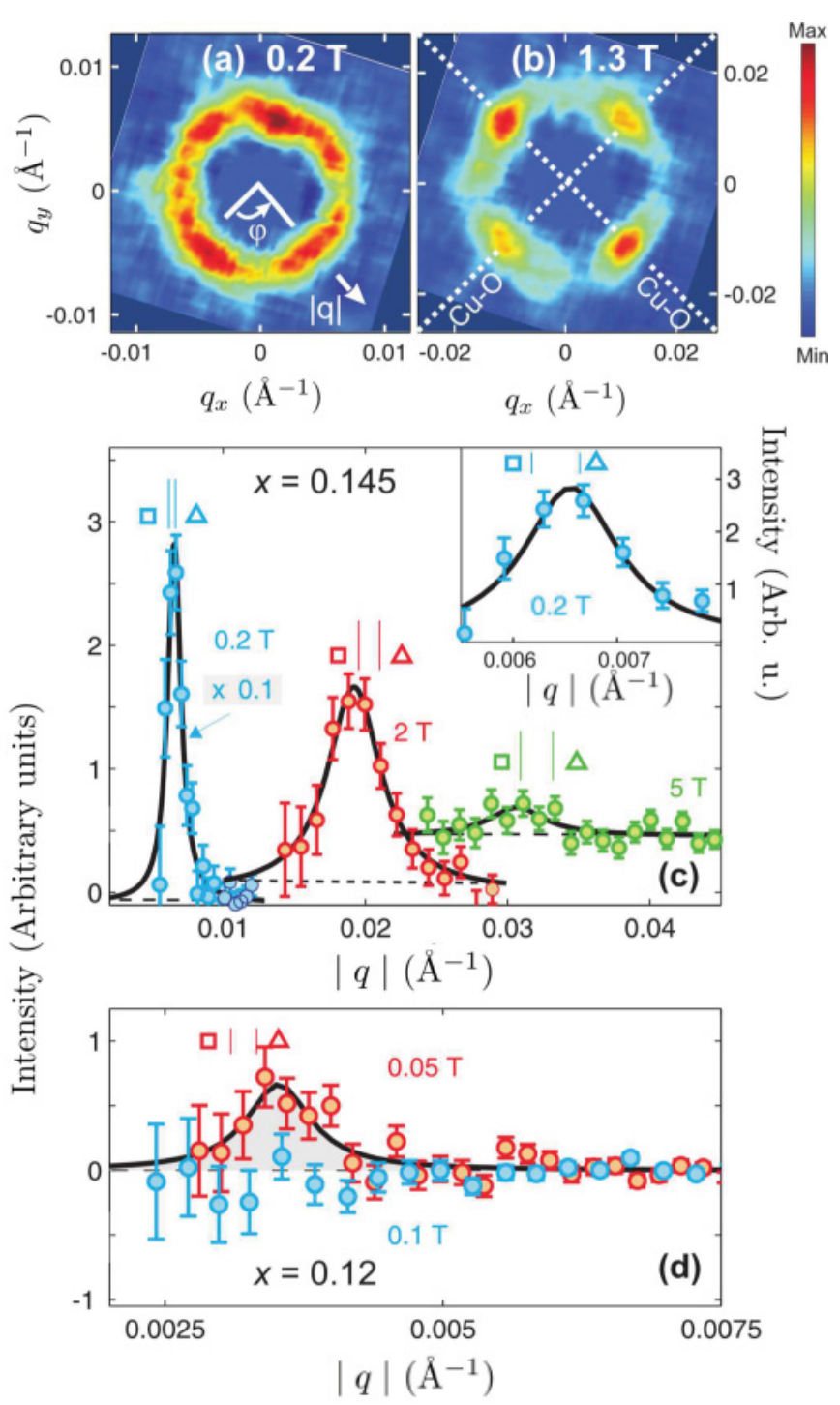

FIG. 3. (Color online) (a), (b) Vortex lattice diffraction patterns, recorded using a position-sensitive detector (see Fig. 2), of LSCO $x=0.145$ under applied magnetic fields of $0.2 \mathrm{~T}$ and $1.3 \mathrm{~T}$, respectively. Notice that the $\mathrm{Cu}-\mathrm{O}$ axes are along the diagonal. (c), (d) Azimuthally averaged momentum $|q|$ dependence of the scattered intensity, summed over rocking angles, for dopings $x=$ 0.145 and 0.12 , and applied magnetic fields as indicated. For visibility, the $0.2 \mathrm{~T}$ data in (c) have been divided by a factor of ten. Inset of (c) is a zoom on the $0.2 \mathrm{~T}$ data. The vertical bars above the diffraction peaks indicate the expected positions for square and regular hexagonal VL coordinations. The solid black lines in (c) and (d) are Lorentzian fits to the data.

diffracted intensity measured in LSCO $x=0.145$ at $0.2 \mathrm{~T}$ does not show well-defined Bragg spots. This indicates a large $\tau_{\mathrm{A}}$ and poor orientational order of the VL about the $c$ axis. Nevertheless we can determine the VL coordination by averaging over the azimuthal angle $\varphi$ so that the diffracted intensity $I(|\mathbf{q}|)$ becomes a function of $|\mathbf{q}|$ only [see Fig. 3(c)]. $|\mathbf{G}|$ is determined from the peak position in the $|\mathbf{q}|$ dependence. Fitting a Lorentzian line shape to these $0.2 \mathrm{~T}$ data, yields $\sigma=0.88(2)$ very close to the value expected for a hexagonal VL. At $\mu_{0} H=1.3 \mathrm{~T}$ in Fig. 3(b), we find four Bragg spots with
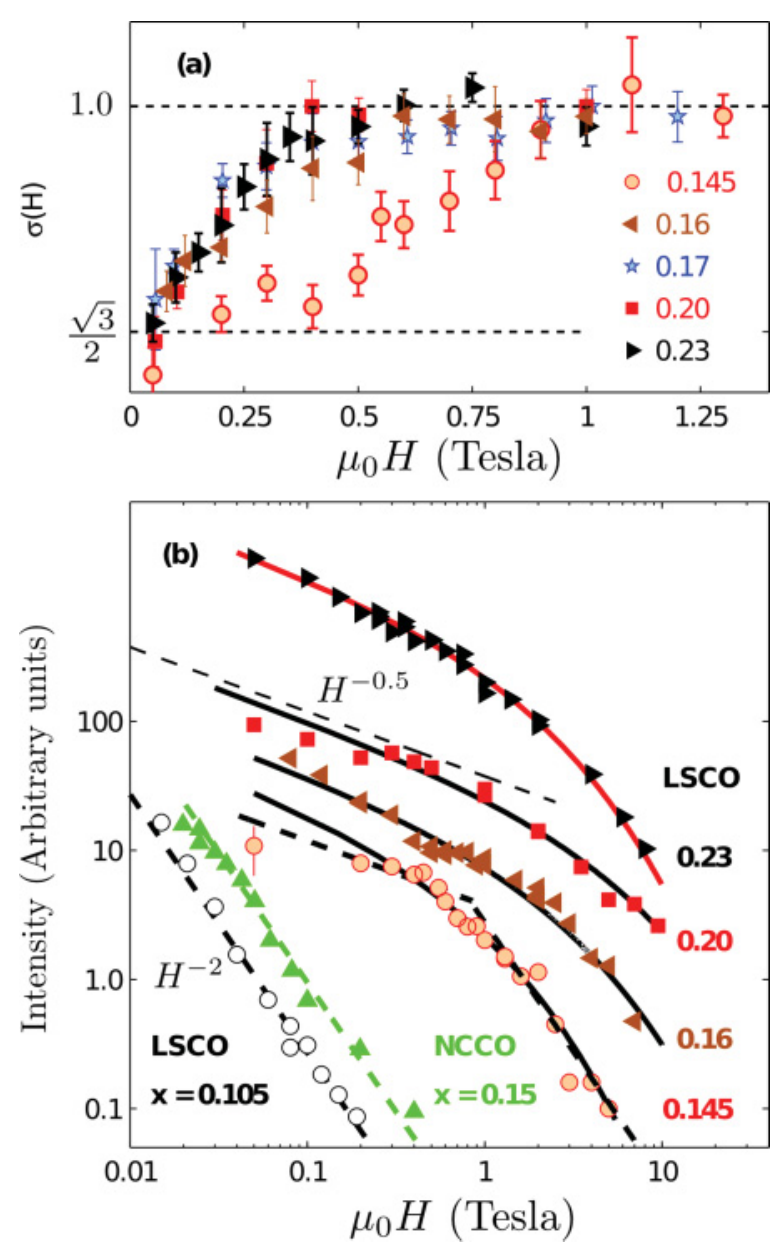

FIG. 4. (Color online) (a) Dimensionless constant $\sigma$, defined in the text, as a function of magnetic field for LSCO $x=0.145,0.16$ 0.17 (Ref. 22), 0.20 (Ref. 23), and 0.23. $\sigma=\sqrt{3} / 2$ is expected for a hexagonal lattice and a square vortex lattice has $\sigma=1$. The change form $\sigma=\sqrt{3} / 2$ to 1 therefore reveals the a hexagonal-tosquare transition of the vortex lattice structure (see also Fig. 1). (b) SANS intensity $I$ versus applied magnetic field for NCCO $x=0.15$ (Ref. 53) and $\mathrm{La}_{2-x} \mathrm{Sr}_{x} \mathrm{CuO}_{4}$ with $x=0.105$ (Ref. 54), and $0.145-0.23$, (this work). For clarity, the intensities for each of the compositions have been given an arbitrary vertical offset. Solid lines are fits to the Clem model form factor where the superconducting coherence length $\xi$ is the only parameter (see Fig. 5). Dashed lines indicate power-law dependencies; $I \sim H^{-0.5}$ and $I \sim H^{-2}$. Notice that the Bragg glass paradigm for vortices in the presence of disorder is consistent with a crossover from $I \sim H^{-0.5}$ to $I \sim H^{-2}$ (see text).

G along the $\mathrm{Cu}-\mathrm{O}$ bond directions and $\sigma=0.99(1)$, indicating not only an improved VL orientational order but moreover a square coordination. As shown quantitatively in Fig. 4(a), the VL coordination in underdoped LSCO $x=0.145$ changes steadily from hexagonal to square over the range $\mu_{0} H=0.2$ to $0.8 \mathrm{~T}$. In contrast, on the optimally doped and overdoped side of the phase diagram $(x \geqslant 0.17)$, the VL structure becomes square by $\mu_{0} H \approx 0.4 \mathrm{~T}$ [Fig. 4(a)].

A minimum in either the Fermi velocity $v_{F}(\mathbf{k})$ or the superconducting gap $\Delta(\mathbf{k})$ are well-known sources of fielddriven hexagonal-to-square VL transitions. ${ }^{37-40}$ In both LSCO and $\mathrm{YBa}_{2} \mathrm{Cu}_{3} \mathrm{O}_{y}(\mathrm{YBCO})$ the band structure is predominantly two dimensional but with some $c$-axis dispersion near the 
$\mathbf{k}=(\pi, 0)$ point $^{41,42}$ It was previously suggested that the VL morphology should be understood from the Fermi surface topology near the $(\pi, 0)$ point. ${ }^{39}$ A crucial difference between the band structures of LSCO and YBCO is that in LSCO, a Van Hove singularity crosses the Fermi level $\left(\epsilon_{F}\right)$ at $(\pi, 0)$ somewhere between $x=0.17$ and $0.22,{ }^{42,43}$ leading to a huge $v_{F}$ anisotropy. This offers an explanation as to why the square VL is oriented along the $(\pi, 0)$ direction in LSCO as opposed to the nodal $(\pi, \pi)$ direction in YBCO. ${ }^{37-39,44-46}$ As LSCO is underdoped, the Van Hove singularity is pushed further away from $\epsilon_{F},{ }^{43}$ leading to a smaller $v_{F}$ anisotropy and consequently a larger field is required to form the square $\mathrm{VL}$, as indeed observed for LSCO $x=0.145$ [Fig. 4(a)].

\section{B. Diffracted small-angle neutron scattering intensity}

We define the VLintensity of the first-order diffraction peak $I$ as the sum of the area under $I(|\mathbf{q}|)$, which is itself a sum over rocking angles. Overlap measurements of $I$ vs $\mu_{0} H$, shown in Fig. 4(b) for $x=0.105-0.23$, were done whenever the neutron wavelength $\lambda_{n}$ was changed. For $x \geqslant 0.16$, intensity could be observed up to the highest applied field $10 \mathrm{~T}$. By contrast, for $x=0.145$ no intensity was observed above the quantum critical field $\left(\mu_{0} H=7 \pm 1 \mathrm{~T}\right.$ in our sample) for SDW order. ${ }^{26,27,31}$ It is, however, still possible that the VL extends slightly into the SDW-ordered phase. This is the case in LSCO $x=0.105,{ }^{33}$ where a three-dimensional (3D) vortex lattice exhibits $I \propto H^{-2}$ [Fig. 4(b)] over two decades of intensity and coexists with short-range SDW order ${ }^{26}$ at very low fields $H_{c 1}<\mu_{0} H \lesssim 0.2 \mathrm{~T}$. VL intensity is also observed in LSCO $x=0.12$; a compound where long-range SDW order exists already in zero field. ${ }^{26}$ At $\mu_{0} H=0.05 \mathrm{~T}$, the $|\mathbf{q}|$ dependence of the intensity $I(|\mathbf{q}|)$ [Fig. 3(d)] suggests a hexagonal VL coordination. This provides evidence by SANS of a VL coexisting with SDW order at the 1/8 anomaly. The field range of coexistence is small; on increasing the field to just $\mu_{0} H=0.1 \mathrm{~T}$, Fig. 3(d) shows that the VL signal has already fallen to the background level. Notice that the fields 0.1-0.2 $\mathrm{T}$ are much smaller than those required to decouple 3D superconductivity. ${ }^{47}$

\section{Coherence length from the vortex lattice form factor}

In the case of perfect crystalline VL order, the observed intensity of the first-order diffraction peak $I(H) \propto \sum \mathcal{F}^{2} /|\mathbf{G}|$ where $\mathcal{F}$ is the form factor of a single vortex and has units of field. The sum is over all the $\mathbf{q}$ vectors contributing to the intensity near wave vector $|\mathbf{G}|$, and we have assumed that the rocking curve width remains constant with field in obtaining $I(H)$. A variational solution to the Ginzburg-Landau model, namely the Clem model for the VL form factor, ${ }^{48}$ yields $\mathcal{F} \propto G K_{1}(G \xi)$, where $K_{1}$ denotes the modified Bessel function of first order, $G=|\mathbf{G}|=2 \pi \sqrt{H / \sigma \Phi_{0}}$, and the vortex core size $\xi$ is the only fit parameter. ${ }^{49}$ We point out that the application of the Clem model to our data yields an upper bound for $\xi$; disorder effects are, for example, not included. ${ }^{50}$ With increasing vortex lattice disorder, the degree by which the Clem model overestimates the coherence length is larger. By comparing the doping dependence of the extracted $\xi$ with the Ginzburg-Landau coherence length
$\xi_{G L}=\sqrt{\Phi_{0} / 2 \pi H_{c 2}}$ estimated indirectly from specific heat ${ }^{51}$ and high-field magnetoresistance experiments, ${ }^{52}$ a reasonable agreement is found on the overdoped side (see Fig. 5). This suggests that our SANS data indeed provide a measure of $\xi$, even though disorder is undoubtedly present. Identifying $\xi$ with the Pippard coherence length $\xi_{p} \sim \hbar v_{F}(k) / \Delta(k)$ suggests that the relatively short coherence length $\xi$ around optimally doped LSCO is not only due to the large pairing gap $\Delta ;$ the small Fermi velocity is also playing a significant role.

\section{Disorder effects and structure factor}

On the underdoped side, we find a strong discrepancy between the coherence length estimated from specific heat and the SANS data fitted with the Clem model for the form factor (see Fig. 5). For LSCO $x=0.145$, we find $\xi \sim 80 \AA$ corresponding to an unrealistically small upper critical field $\mu_{0} H_{c 2}=\Phi_{0} / 2 \pi \xi^{2} \sim 5$ T. A larger coherence length may result from the weakening of superconductivity due to competition with, for example, magnetism. However, this does not explain the discrepancy between our SANS data and the specific heat data. ${ }^{51} \mathrm{~A}$ more plausible explanation is that the VL disorder potential increases with underdoping. It is possible that VL disorder proliferates as the system is tuned toward the state where magnetism and superconductivity coexist.

The VL displacements throughout the doping range are well described by elastic theory, namely the Bragg glass (BrG) paradigm. ${ }^{16,17}$ In the presence of disorder, the positional order of an elastic VL decays exponentially with a characteristic length scale $R_{A}$. (With increasing disorder $R_{A} \rightarrow 0$.) If such an exponential decay were to persist at all length scales $R$, a total destruction of long-range order would result. ${ }^{55}$ This

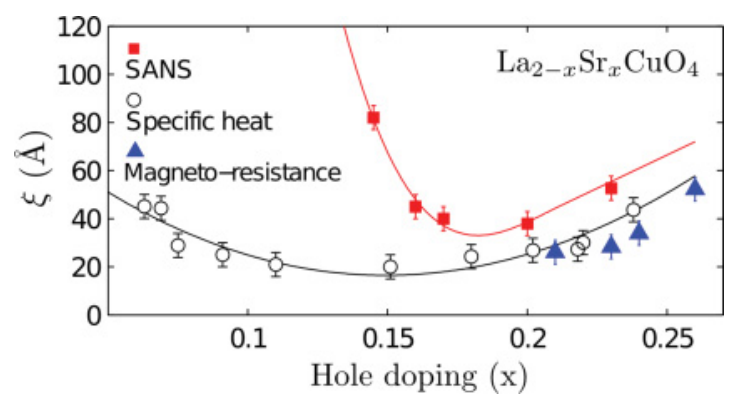

FIG. 5. (Color online) Superconducting coherence length $\xi$ in LSCO extracted from magnetoresistance (solid blue points), ${ }^{52}$ specific heat (open black points), ${ }^{51}$ and our SANS measurements (solid red points) and plotted as a function of the hole concentration $x$. The high-field magnetoresistance study measures the upper critical field $H_{c 2}$ at low temperatures and we used $H_{c 2}=\Phi_{0} /\left(2 \pi \xi^{2}\right)$ to estimate the coherence length $\xi$. On the other hand, the specific heat and the SANS experiments were carried out at fields smaller or comparable to $H_{c 2}$. To extract the superconducting coherence length from the SANS data we used the Clem model for the VL form factor. Notice that in presence of vortex lattice disorder, the Clem model will overestimate the coherence length (see text). This may explain why the coherence length extracted from the SANS data lies systematically above the specific heat and magnetoresistance measurements. All lines are guides to the eye. 
proposed destruction, even under weak disorder, presented a longstanding puzzle with respect to experimental observations where Bragg peaks may readily be observed. Theoretically, the puzzle was resolved with the advent of the $\mathrm{BrG}$ paradigm, in which an asymptotic regime for $R>R_{A}$ enters, where the positional order decays only weakly, leading to algebraically diverging Bragg peaks. At the crossover scale $R_{A}$ vortex displacements are comparable to the lattice spacing $a_{0}=$ $\sqrt{\sigma \Phi_{0} / H}$. In earlier muon spin rotation $(\mu \mathrm{SR})$ work on a LSCO $x=0.105$ sample, an order-disorder transition was observed and associated with a transition out of the quasilong-range-ordered BrG phase. ${ }^{33}$ VL correlations in the $\mathrm{BrG}$ phase have been explored more directly in a study of low-purity niobium. ${ }^{21}$ We point out that the BrG paradigm can explain both the $H^{-0.5}$ and $H^{-2}$ field dependencies of intensity [c.f. Fig. 4(b)], as well as the crossover between them. Dependent on whether the instrumental resolution $s \approx 60 a_{0}$ is larger or smaller than $R_{A}$, a different field dependence of the SANS intensity is predicted. ${ }^{56}$ For $s<R_{A}$, the contribution to the structure factor is identical to that of a crystalline VL, hence $I \sim 1 / \sqrt{H}$. In the other limit $s>R_{A}$, an additional factor $H^{-\mu}$ contributes to the intensity. Elastic theory ${ }^{16}$ yields $\mu=$ $3 / 2$ and hence $I \approx H^{-2}$-as indeed we observed previously in LSCO $x=0.105$ [see Fig. 4(b)]. ${ }^{54}$ The intensity for $x=0.145$ is also consistent with a $H^{-0.5}$ to $H^{-2}$ crossover (see Fig. 3). At the crossover field $(\sim 0.85 \mathrm{~T}), R_{A} \approx s \approx 60 a_{0} \approx 3 \mu \mathrm{m}$. By contrast, in LSCO $x=0.105$ the crossover field $\ll 0.05 \mathrm{~T}$, implying that the disorder potential increases dramatically with decreasing doping. This disordering seems to occur as the static magnetic correlation length $\zeta$ approaches the VL spacing $\left(\zeta \rightarrow a_{0}\right)$, suggesting an electronic origin to the VL disorder effectuated by real-space competition between $\zeta$ and $a_{0}$, rather than orthorhombic twin boundaries or impurities as observed in other superconductors. ${ }^{21}$ We noticed that the BrG model also provides an excellent description for the field dependence of intensity in $\mathrm{Nd}_{2-x} \mathrm{Ce}_{x} \mathrm{CuO}_{4}$ (NCCO) with $x=0.15$ [Fig. 4(b)]. ${ }^{53}$ More experimental studies are required on NCCO to establish if the VL disorder therein has origins similar to that in LSCO.

\section{E. Real-space picture}

We now consider how magnetic and superconducting order parameters might coexist in real space. Around $x=0.12$ doping in LSCO, $\mu \mathrm{SR}$ measurements revealed magnetic and superconducting fractions that together exceed $100 \%$. It was therefore concluded that the magnetic and superconducting order parameters are not phase separated but rather intertwined on a nanometer scale. ${ }^{57,58}$ This real-space picture is not easily reconciled with neutron diffraction studies that report a magnetic correlation length of several hundreds of angstroms. ${ }^{26,27,29,30}$ One possibility is that the charge of the muon induces magnetism in which case the $\mu \mathrm{SR}$ technique overestimates the magnetic volume fraction. ${ }^{59}$ Here we showed that the vortex lattice becomes more disordered as the vortex interspacing approaches the magnetic correlation length. This suggests that the magnetic and superconducting order parameters are coupled. How magnetism, superconductivity, and vortices are arranged in real space when the spin correlation length is larger than the vortex spacing $\left(\zeta>a_{0}\right)$ is an interesting question that is difficult to address with the SANS technique since no observable SANS signal is found in that region of the phase diagram (see Fig. 1). Direct imaging techniques ${ }^{18-20}$ are undoubtedly more informative in this regime although it may be experimentally challenging to probe magnetism and vortices simultaneously.

\section{CONCLUSION}

In summary, our studies of the vortex lattice in LSCO allow us to draw two main conclusions. First, near optimal doping, and far from the SDW instability, the VL structure/orientation and the small superconducting coherence length $\xi$ (and hence large upper critical field $H_{c 2}$ ) may both be rationalized as arising from a vanishing Fermi velocity due to the Van Hove singularity near the zone boundary. Second, we find that the fermiological picture breaks down as the SDW instability is approached by tuning either the doping or the applied magnetic field. There, the vortex lattice structure factor needs to be accounted for in the field dependence of the observable SANS intensity. The Bragg glass paradigm, describing vortex lattices in the presence of weak disorder, accounts for the SANS intensity behavior across the entire phase diagram (where a SANS signal is discernible), from the underdoped $x=0.105$, to the optimally doped $x \sim 0.16$, and overdoped $x=0.23$ regimes. In particular, it is able to explain the crossover as the SDW instability is approached. Evidently, the SDW order acts as an electronic provenience of disorder on the vortex lattice in LSCO.

\section{ACKNOWLEDGMENTS}

Experiments were performed at the Institut Laue-Langevin (ILL), Grenoble, France and at the Swiss Spallation Source SINQ, Paul Scherrer Institute, Villigen, Switzerland. We acknowledge discussions with C. Niedermayer, B. M. Andersen, and N. B. Christensen and support from the Swiss National Science Foundation (through National Centres of Competence in Research, Materials with Novel Electronic Properties, and Grants No. 200020-105151, and No. PBEZP2-122855), the Danish Natural Science Research Council under DanScatt, the UK Engineering and Physical Sciences Research Council, and by the Ministry of Education and Science of Japan.
${ }^{1}$ Paul C. Canfield, Peter L. Gammel, and David J. Bishop, Phys. Today 51, 40 (1998).

${ }^{2}$ K.-H. Müller and V. N. Narozhnyi, Rep. Prog. Phys. 64, 943 (2001).
${ }^{3}$ L. C. Gupta, Adv. Phys. 55, 691 (2006).

${ }^{4}$ Sergey L. Budko, and Paul C. Canfield, C. R. Phys. 7, 56 (2006). 
${ }^{5}$ B. Lake, H. M. Rønnow, N. B. Christensen, G. Aeppli, K. Lefmann, D. F. McMorrow, P. Vorderwisch, P. Smeibidl, N. Mangkorntong, T. Sasagawa, M. Nohara, H. Takagi, and T. E. Mason, Nature (London) 415, 299 (2002).

${ }^{6}$ M. R. Eskildsen, E. M. Forgan, and H. Kawano-Furukawa, Rep. Prog. Phys. 74, 124504 (2011).

${ }^{7}$ P. Monthoux, D. Pines, and G. G. Lonzarich, Nature (London) 450, 1177 (2007).

${ }^{8}$ D. Jérome, J. Magn. Magn. Mater. 3134, 2028 (1983).

${ }^{9}$ Matthias Vojta, Adv. Phys. 58, 699 (2009).

${ }^{10}$ Eugene Demler, Subir Sachdev, and Ying Zhang, Phys. Rev. Lett. 87, 067202 (2001).

${ }^{11}$ J. Hoffmann, E. W. Hudson, K. M. Lang, V. Madhavan, H. Eisaki, S. Uchida, and J. C. Davis, Science 295, 466 (2002).

${ }^{12}$ Subir Sachdev, Rev. Mod. Phys. 75, 913 (2003).

${ }^{13}$ B. M. Andersen, O. F. Syljuasen, and P. Hedegard, Phys. Rev. B 80, 052509 (2009).

${ }^{14}$ B. Lake, G. Aeppli, K. N. Clausen, D. F. McMorrow, K. Lefmann, N. E. Hussey, N. Mangkorntong, M. Nohara, H. Takagi, T. E. Mason, and A. Schröder, Science 291, 1759 (2001).

${ }^{15}$ A. Shekhter, L. N. Bulaevskii, and C. D. Batista, Phys. Rev. Lett. 106, 037001 (2011).

${ }^{16}$ T. Giamarchi and P. Le Doussal, Phys. Rev. B 52, 1242 (1995).

${ }^{17}$ S. Bogner, T. Emig, and T. Nattermann, Phys. Rev. B 63, 174501 (2001).

${ }^{18}$ L. Ya. Vinnikov, J. Anderegg, S. L. Budâko, P. C. Canfield, and V. G. Kogan, Phys. Rev. B 71, 224513 (2005).

${ }^{19}$ Hendrik Bluhm, Suchitra E. Sebastian, Janice W. Guikema, I. R. Fisher, and Kathryn A. Moler, Phys. Rev. B 73, 014514 (2006).

${ }^{20}$ B. Kalisky, J. R. Kirtley, J. G. Analytis, J.-H. Chu, I. R. Fisher, and K. A. Moler, Phys. Rev. B 83, 064511 (2011).

${ }^{21}$ M. Laver, E. M. Forgan, A. B. Abrahamsen, C. Bowell, Th. Geue, and R. Cubitt, Phys. Rev. Lett. 100, 107001 (2008).

${ }^{22}$ R. Gilardi, J. Mesot, A. Drew, U. Divakar, S. L. Lee, E. M. Forgan, O. Zaharko, K. Conder, V. K. Aswal, C. D. Dewhurst, R. Cubitt, N. Momono, and M. Oda, Phys. Rev. Lett. 88, 217003 (2002).

${ }^{23}$ R. Gilardi, J. Mesot, A. J. Drew, U. Divakar, S. L. Lee, N. H. Andersen, J. Kohlbrecher, N. Momono, and M. Oda, Physica C 408-410, 491 (2004).

${ }^{24}$ J. Chang, J. Mesot, R. Gilardi, J. Kohlbrecher, A. J. Drew, U. Divakar, S. J. Lister, S. L. Lee, S. P. Brown, D. Charalambous, E. M. Forgan, C. D. Dewhurst, R. Cubitt, N. Momono, and M. Oda, Physica B 385-386, 35 (2006).

${ }^{25}$ T. Nakano, N. Momono, M. Oda, and M. Ido, J. Phys. Soc. Jpn. 67, 2622 (1998).

${ }^{26}$ J. Chang, Ch. Niedermayer, R. Gilardi, N. B. Christensen, H. M. Rønnow, D. F. McMorrow, M. Ay, J. Stahn, O. Sobolev, A. Hiess, S. Pailhes, C. Baines, N. Momono, M. Oda, M. Ido, and J. Mesot, Phys. Rev. B 78, 104525 (2008).

${ }^{27}$ J. Chang, N. B. Christensen, Ch. Niedermayer, K. Lefmann, H. M. Rø nnow, D. F. McMorrow, A. Schneidewind, P. Link, A. Hiess, M. Boehm, R. Mottl, S. Pailhés, N. Momono, M. Oda, M. Ido, and J. Mesot, Phys. Rev. Lett. 102, 177006 (2009).

${ }^{28}$ O. J. Lipscombe, S. M. Hayden, B. Vignolle, D. F. McMorrow, and T. G. Perring, Phys. Rev. Lett. 99, 067002 (2007).

${ }^{29}$ B. Khaykovich, Y. S. Lee, R. W. Erwin, S.-H. Lee, S. Wakimoto, K. J. Thomas, M. A. Kastner, and R. J. Birgeneau, Phys. Rev. B 66, 014528 (2002).
${ }^{30}$ B. Khaykovich, R. J. Birgeneau, F. C. Chou, R. W. Erwin, M. A. Kastner, S.-H. Lee, Y. S. Lee, P. Smeibidl, P. Vorderwisch, and S. Wakimoto, Phys. Rev. B 67, 054501 (2003).

${ }^{31}$ B. Khaykovich S. Wakimoto, R. J. Birgeneau, M. A. Kastner, Y. S. Lee, P. Smeibidl, P. Vorderwisch, and K. Yamada, Phys. Rev. B 71, 220508(R) (2005).

${ }^{32}$ Susumu Katano, Masugu Sato, Kazuyoshi Yamada, Takao Suzuki, and Tetsuo Fukase, Phys. Rev. B 62, R14677 (2000).

${ }^{33}$ U. Divakar, A. J. Drew, S. L. Lee, R. Gilardi, J. Mesot, F. Y. Ogrin, D. Charalambous, E. M. Forgan, G. I. Menon, N. Momono, M. Oda, C. D. Dewhurst, and C. Baines, Phys. Rev. Lett. 92, 237004 (2004).

${ }^{34}$ M. Kofu, S.-H. Lee, M. Fujita, H.-J. Kang, H. Eisaki, and K. Yamada, Phys. Rev. Lett. 102, 047001 (2009).

${ }^{35}$ D. Haug, V. Hinkov, Y. Sidis, P. Bourges, N. B. Christensen, A. Ivanov, T. Keller, C. T. Lin, and B. Keimer, New J. Phys. 12, 105006 (2010).

${ }^{36}$ Joachim Kohlbrecher and Werner Wagner, J. Appl. Crystallogr. 33, 804 (2000).

${ }^{37}$ B. Keimer, W. Y. Shih, R. W. Erwin, J. W. Lynn, F. Dogan, and I. A. Aksay, Phys. Rev. Lett. 73, 3459 (1994).

${ }^{38}$ S. P. Brown, D. Charalambous, E. C. Jones, E. M. Forgan, P. G. Kealey, A. Erb, and J. Kohlbrecher, Phys. Rev. Lett. 92, 067004 (2004).

${ }^{39}$ N. Nakai, P. Miranovic, M. Ichioka, and K. Machida, Phys. Rev. Lett. 89, 237004 (2002).

${ }^{40}$ M. R. Eskildsen, Charles D. Dewhurst, Bart W. Hoogenboom, Cedomir Petrovic, and Paul C. Canfield, Phys. Rev. Lett. 90, 187001 (2003).

${ }^{41}$ Sudip Chakravarty, Asle Sudbø, Philip W. Anderson, and Steven Strong, Science 261, 337 (1993).

${ }^{42}$ T. Yoshida, X. J. Zhou, K. Tanaka, W. L. Yang, Z. Hussain, Z.-X. Shen, A. Fujimori, S. Sahrakorpi, M. Lindroos, R. S. Markiewicz, A. Bansil, Seiki Komiya, Yoichi Ando, H. Eisaki, T. Kakeshita, and S. Uchida, Phys. Rev. B 74, 224510 (2006).

${ }^{43}$ J. Chang, M. Shi, S. Pailhes, M. Mansson, T. Claesson, O. Tjernberg, A. Bendounan, Y. Sassa, L. Patthey, N. Momono, M. Oda, M. Ido, S. Guerrero, C. Mudry, and J. Mesot, Phys. Rev. B 78, 205103 (2008).

${ }^{44}$ J. S. White, S. P. Brown, E. M. Forgan, M. Laver, C. J. Bowell, R. J. Lycett, D. Charalambous, V. Hinkov, A. Erb, and J. Kohlbrecher, Phys. Rev. B 78, 174513 (2008).

${ }^{45}$ J. S. White, R. W. Heslop, A. T. Holmes, E. M. Forgan, V. Hinkov, N. Egetenmeyer, J. L. Gavilano, M. Laver, C. D. Dewhurst, R. Cubitt, and A. Erb, Phys. Rev. B 84, 104519 (2011).

${ }^{46}$ J. S. White, V. Hinkov, R. W. Heslop, R. J. Lycett, E. M. Forgan, C. Bowell, S. Strassle, A. B. Abrahamsen, M. Laver, C. D. Dewhurst, J. Kohlbrecher, J. L. Gavilano, J. Mesot, B. Keimer, and A. Erb, Phys. Rev. Lett. 102, 097001 (2009).

${ }^{47}$ A. A. Schafgans, A. D. LaForge, S. V. Dordevic, M. M. Qazilbash, W. J. Padilla, K. S. Burch, Z. Q. Li, Seiki Komiya, Yoichi Ando, and D. N. Basov, Phys. Rev. Lett. 104, 157002 (2010).

${ }^{48}$ J. R. Clem, J. Low Temp. Phys. 18, 427 (1975).

${ }^{49}$ In our field range $\mu_{0} H \gtrsim 0.05 \mathrm{~T}, \mathcal{F}(H)$ is practically independent of the superconducting penetration depth $\lambda$. In the Clem model, ${ }^{48}$ the argument $z$ of the modified Bessel function $K_{1}(z)$ is strictly $z=$ $\left(G^{2}+\lambda^{-2}\right)^{1 / 2} \xi$. Here $G \gtrsim 0.003 \AA^{-1}$ and $1 / \lambda \lesssim 0.0005 \AA^{-1},{ }^{60}$ so $z \simeq G \xi$. In the Clem model, $\lambda$ also enters as an $H$-independent prefactor $1 / \lambda K_{1}(\xi / \lambda)$. 
${ }^{50} \mathrm{~A}$ simple model for VL disorder comprises the static Debye-Waller factor $W=\exp \left(-4 \pi^{2}\left\langle u^{2}\right\rangle / a_{0}^{2}\right)$ where $\left\langle u^{2}\right\rangle$ is the root mean square vortex displacement (along q) and $a_{0}=\sqrt{\sigma \Phi_{0} / H}$ is the vortex lattice spacing. Due to the similar field dependence of $\mathcal{F}$ and $W$, it is not possible to disentangle contributions arising from the vortex core size $\xi$ and Debye-Waller effects.

${ }^{51}$ Yue Wang and Hai-Hu Wen, Eur. Phys. Lett. 81, 57007 (2008).

${ }^{52}$ Patrick M. C. Rourke, Ioanna Mouzopoulou, Xiaofeng Xu, Christos Panagopoulos, Yue Wang, Baptiste Vignolle, Cyril Proust, Evgenia V. Kurganova, Uli Zeitler, Yoichi Tanabe, Tadashi Adachi, Yoji Koike, and Nigel E. Hussey, Nature Phys. 7, 455458 (2011).

${ }^{53}$ R. Gilardi, J. Mesot, S. P. Brown, E. M. Forgan, A. Drew, S. L. Lee, R. Cubitt, C. D. Dewhurst, T. Uefuji, and K. Yamada, Phys. Rev. Lett. 93, 217001 (2004).
${ }^{54}$ J. Chang and J. Mesot, PRAMANA-J. Phys. 71, 679 (2008).

${ }^{55}$ A. I. Larkin, Sov. Phys. JETP 31, 784 (1970).

${ }^{56}$ T. Klein, I. Joumard, S. Blanchard, J. Marcus, R. Cubitt, T. Giamarchi, and P. Le Doussal, Nature (London) 413, 404 (2001).

${ }^{57}$ A. T. Savici, Y. Fudamoto, I. M. Gat, T. Ito, M. I. Larkin, Y. J. Uemura, G. M. Luke, K. M. Kojima, Y. S. Lee, M. A. Kastner, R. J. Birgeneau, and K. Yamada, Phys. Rev. B 66, 014524 (2002).

${ }^{58}$ Y. J. Uemura, Solid State Commun. 126, 23 (2003).

${ }^{59}$ Hung T. Dang, Emanuel Gull, and Andrew J. Millis, Phys. Rev. B 81, 235124 (2010).

${ }^{60}$ C. Panagopoulos, B. D. Rainford, J. R. Cooper, W. Lo, J. L. Tallon, J. W. Loram, and J. Betouras, Y. S. Wang, and C. W. Chu, Phys. Rev. B 60, 14617 (1999). 